\title{
Probing New Physics with Leptonic Rare B Decays
}

\section{Robert Fleischer*}

Nikhef, Science Park 105, NL-1098 XG Amsterdam, Netherlands

Faculty of Science, Vrije Universiteit Amsterdam, NL-1081 HV Amsterdam, Netherlands

E-mail: Robert.Fleischer@nikhef.nl

Leptonic rare decays of neutral B mesons belong to the most favourable processes for testing the flavour structure of the Standard Model, with an outstanding sensitivity to possible new (pseudo)scalar contributions. We discuss implications of the current data for the theoretical description of the flavour sector, discuss the use of new observables to probe New Physics, and make a comparison with recent theoretical studies of leptonic decays of charged B mesons.

Corfu Summer Institute 2019 "School and Workshops on Elementary Particle Physics and Gravity" (CORFU2019)

31 August - 25 September 2019

Corfù, Greece

${ }^{*}$ Speaker. 


\section{Introduction}

In the following discussion, the physics potential of leptonic $B$-meson decays into final states with different lepton flavours $\ell=e, \mu, \tau$ is discussed. Leptonic $B_{q}^{0} \rightarrow \ell^{+} \ell^{-}$decays [1,2] of neutral $B_{q}^{0}$ mesons $(q=s, d)$ are caused by flavour-changing neutral current processes (FCNC). On the other hand, leptonic $B^{-} \rightarrow \ell^{-} \bar{v}_{\ell}$ decays [3] of charged $B$ mesons originate from charged-current interactions which arise at the tree level in the Standard Model (SM).
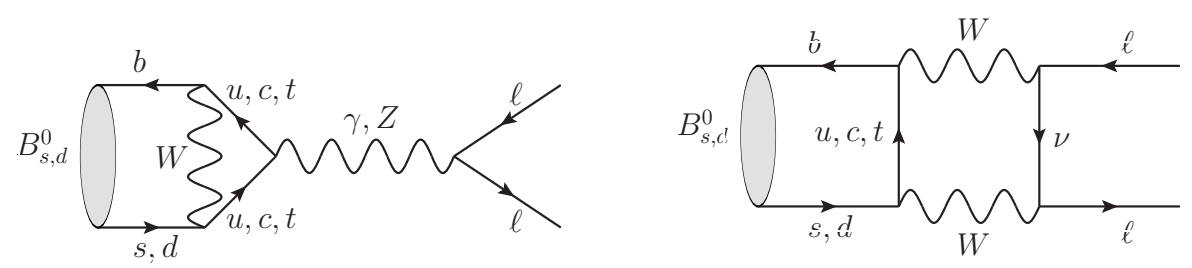

Figure 1: SM contributions to $B_{s, d}^{0} \rightarrow \ell^{+} \ell^{-}$decays: penguin (left) and box (right) topologies.
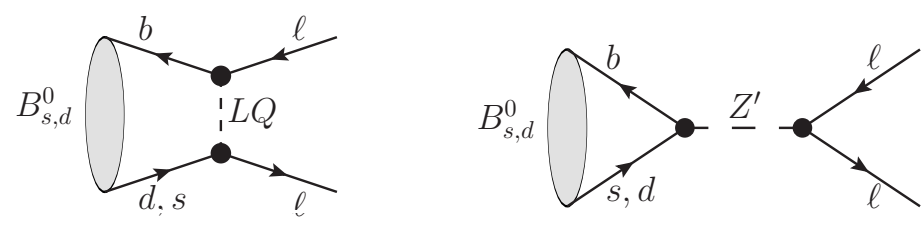

Figure 2: Examples of NP contributions to $B_{s, d}^{0} \rightarrow \ell^{+} \ell^{-}$modes in scenarios with leptoquarks (left) and extra $Z^{\prime}$ bosons (right).

Let us focus on the neutral channels first. In the SM, the FCNCs giving rise to the leptonic $B_{q}^{0} \rightarrow \ell^{+} \ell^{-}$modes originate only from loop contributions through penguin and box topologies, as illustrated in Fig. 1, and lead to a helicity suppression which results in branching ratios proportional to $m_{\ell}^{2}$, where $m_{\ell}$ denotes the masses of the final state leptons. Another key feature is the simple situation concerning strong interactions, which are encoded in the hadronic matrix element

$$
\left\langle 0\left|\bar{b} \gamma_{5} \gamma_{\mu} q\right| B_{q}^{0}(p)\right\rangle=i f_{B_{q}} p_{\mu}
$$

and described by a single non-perturbative parameter, the $B_{q}$ decay constant $f_{B_{q}}$. The $B_{q}^{0} \rightarrow \ell^{+} \ell^{-}$ modes belong to the cleanest rare $B$ decays and offer an outstanding setting to explore the flavour sector of the SM, with high sensitivity to New Physics (NP) contributions, such as new amplitudes arising in models with leptoquarks and $Z^{\prime}$ bosons (see Fig. 2). Particularly interesting are new (pseudo)-scalars, which may lift the helicity suppression. In Fig. 3, we show a compilation of experimental information in comparison with the SM picture. So far, only $B_{s}^{0} \rightarrow \mu^{+} \mu^{-}$has been observed, which was a highlight of LHC run 1 . In the case of $B_{s, d}^{0} \rightarrow \tau^{+} \tau^{-}$, the helicity suppression is not very effective due to the large $\tau$ mass but the $\tau$ reconstruction makes experimental analyses challenging. Interestingly, the $B_{s, d} \rightarrow e^{+} e^{-}$modes, which are extremely helicity suppressed in the $\mathrm{SM}$, have not yet received attention at the LHC (until very recently as we shall discuss below).

New observables of the decay $B_{s} \rightarrow \mu^{+} \mu^{-}$were pointed out, which offer interesting probes at the high-precision frontier [4, 5]. Using the available experimental $B_{s}^{0} \rightarrow \mu^{+} \mu^{-}$data obtained at the LHC as a guideline, let us address the following questions: 

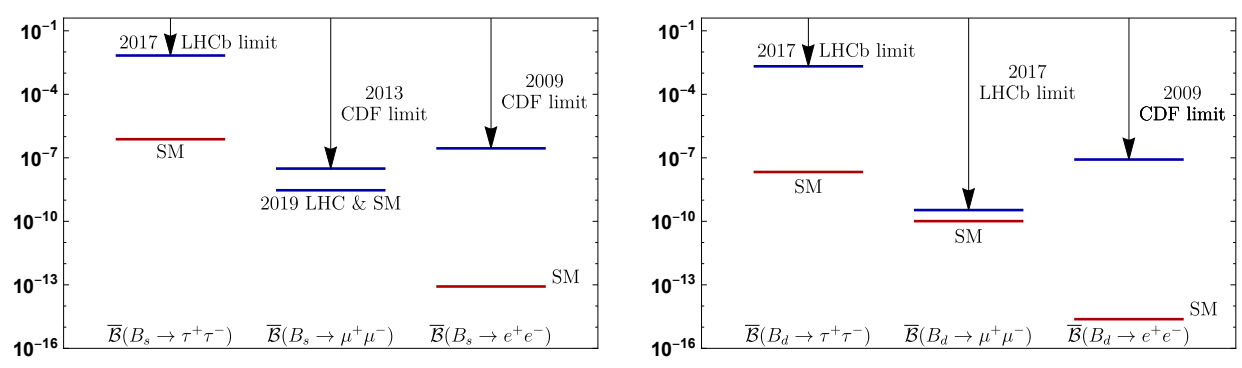

Figure 3: Overview of the experimental information on $B_{q} \rightarrow \ell^{+} \ell^{-}$branching ratios as defined in Eq. (3.2) and comparison with the corresponding SM predictions.

- What are the constraints on NP contributions [1]?

- How large could the $B_{s, d}^{0} \rightarrow \tau^{+} \tau^{-}, B_{s, d}^{0} \rightarrow e^{+} e^{-}$branching ratios actually be [1]?

- What is the impact of possible new sources of CP violation [2]?

The physics potential underlying these questions can be revealed in analyses of the $B_{s, d}^{0} \rightarrow \ell^{+} \ell^{-}$ modes at the future high-precision frontier.

\section{Theoretical Framework}

The low-energy effective Hamiltonian for $\bar{B}_{q}^{0} \rightarrow \ell^{+} \ell^{-}$modes can be written as follows [1]:

$$
\mathscr{H}_{\mathrm{eff}}=-\frac{G_{\mathrm{F}}}{\sqrt{2} \pi} V_{t q}^{*} V_{t b} \alpha\left[C_{10}^{q, \ell \ell} O_{10}+C_{S}^{q, \ell \ell} O_{S}+C_{P}^{q, \ell \ell} O_{P}+C_{10}^{q, \ell \ell^{\prime}} O_{10}^{\prime}+C_{S}^{q, \ell \ell^{\prime}} O_{S}^{\prime}+C_{P}^{q, \ell \ell^{\prime}} O_{P}^{\prime}\right] .
$$

Here the short-distance physics is described by the Wilson coefficients $C_{i}^{q, \ell \ell}, C_{i}^{q, \ell^{\prime}}$ of the fourfermion operators

$$
\begin{aligned}
O_{10} & =\left(\bar{q} \gamma_{\mu} P_{L} b\right)\left(\bar{\ell} \gamma^{\mu} \gamma_{5} \ell\right), & O_{10}^{\prime} & =\left(\bar{q} \gamma_{\mu} P_{R} b\right)\left(\bar{\ell} \gamma^{\mu} \gamma_{5} \ell\right), \\
O_{S} & =m_{b}\left(\bar{q} P_{R} b\right)(\bar{\ell} \ell), & O_{S}^{\prime} & =m_{b}\left(\bar{q} P_{L} b\right)(\bar{\ell} \ell), \\
O_{P} & =m_{b}\left(\bar{q} P_{R} b\right)\left(\bar{\ell} \gamma_{5} \ell\right), & O_{P}^{\prime} & =m_{b}\left(\bar{q} P_{L} b\right)\left(\bar{\ell} \gamma_{5} \ell\right),
\end{aligned}
$$

where $P_{L, R} \equiv\left(1 \mp \gamma_{5}\right) / 2, m_{b}$ is the $b$-quark mass, and the $O_{i}^{\prime}$ are obtained from the $O_{i}$ through the replacements $P_{L} \leftrightarrow P_{R}$. The matrix corresponding elements can be expressed in terms of the $B_{q^{-}}$ meson decay constant $f_{B_{q}}$ introduced in Eq. (1.1). In the SM, only the $O_{10}$ operator is present with a real Wilson coefficient $C_{10}^{\mathrm{SM}}$ which governs the SM predictions in Fig. 3. An outstanding feature of $\bar{B}_{s}^{0} \rightarrow \mu^{+} \mu^{-}$with respect to probing NP is the sensitivity to the (pseudo-)scalar lepton densities entering the $O_{(P) S}$ and $O_{(P) S}^{\prime}$ operators.

For the following considerations, it is useful to introduce the combinations of Wilson coefficient functions

$$
\begin{gathered}
P_{\ell \ell}^{q} \equiv\left|P_{\ell \ell}^{q}\right| e^{i \varphi_{P_{q}}^{\ell \ell}} \equiv \frac{C_{10}^{q, \ell \ell}-C_{10}^{q, \ell \ell^{\prime}}}{C_{10}^{\mathrm{SM}}}+\frac{M_{B_{q}}^{2}}{2 m_{\ell}}\left(\frac{m_{b}}{m_{b}+m_{q}}\right)\left[\frac{C_{P}^{q, \ell \ell}-C_{P}^{q, \ell \ell^{\prime}}}{C_{10}^{\mathrm{SM}}}\right] \\
S_{\ell \ell}^{q} \equiv\left|S_{\ell \ell}^{q}\right| e^{i \varphi_{S_{q}}^{\ell \ell}} \equiv \sqrt{1-4 \frac{m_{\ell}^{2}}{M_{B_{q}}^{2}}} \frac{M_{B_{q}}^{2}}{2 m_{\ell}}\left(\frac{m_{b}}{m_{b}+m_{q}}\right)\left[\frac{C_{S}^{q, \ell \ell}-C_{S}^{q, \ell \ell^{\prime}}}{C_{10}^{\mathrm{SM}}}\right],
\end{gathered}
$$


where $\varphi_{P_{q}}^{\ell \ell}$ and $\varphi_{S_{q}}^{\ell \ell}$ are CP-violating phases. The quantities in Eqs. (2.3) and (2.4) were introduced in such a way that we obtain the simple SM relations

$$
\left.P_{\ell \ell}^{q}\right|_{\mathrm{SM}}=1, \quad S_{\ell \ell}^{q} \mid \mathrm{SM}=0 .
$$

\section{In Pursuit of New Physics}

Due to the presence of $B_{s}^{0}-\bar{B}_{s}^{0}$ mixing and the sizeable $B_{s}$ decay width difference

$$
y_{s} \equiv \frac{\Delta \Gamma_{s} \tau_{B_{s}}}{2}=0.0645 \pm 0.0045 \text {, }
$$

a subtle difference arises between the untagged, time-integrated branching ratio

$$
\overline{\mathscr{B}}\left(B_{s} \rightarrow \mu^{+} \mu^{-}\right) \equiv \frac{1}{2} \int_{0}^{\infty}\left\langle\Gamma\left(B_{s}(t) \rightarrow \mu^{+} \mu^{-}\right)\right\rangle d t \stackrel{\text { LHC }}{=}(2.9 \pm 0.4) \times 10^{-9}
$$

measured at the LHC, and the theoretical predictions $\mathscr{B}\left(B_{s} \rightarrow \mu^{+} \mu^{-}\right)$, which usually refer to a setting without the oscillations $[4,6]$. The conversion involves the observable

$$
\mathscr{A}_{\Delta \Gamma_{s}}^{\mu \mu}=\frac{\left|P_{\mu \mu}^{s}\right|^{2} \cos \left(2 \varphi_{P_{s}}^{\mu \mu}-\phi_{s}^{\mathrm{NP}}\right)-\left|S_{\mu \mu}^{s}\right|^{2} \cos \left(2 \varphi_{S_{s}}^{\mu \mu}-\phi_{s}^{\mathrm{NP}}\right)}{\left|P_{\mu \mu}^{s}\right|^{2}+\left|S_{\mu \mu}^{s}\right|^{2}},
$$

where $\phi_{s}^{\mathrm{NP}}$ denotes a possible NP phase of $B_{s}^{0}-\bar{B}_{s}^{0}$ mixing, which is constrained through CP violation in $B_{s}^{0} \rightarrow J / \psi \phi$ decays at the few degree level [7]. In the SM, $\mathscr{A}_{\Delta \Gamma_{s}}^{\mu \mu}$ takes the value +1 , yielding $\overline{\mathscr{B}}\left(B_{s} \rightarrow \mu^{+} \mu^{-}\right)_{\mathrm{SM}}=(3.57 \pm 0.16) \times 10^{-9}$ [1]. Electromagnetic corrections to this quantity were recently calculated and found to be tiny [8]. The observable $\mathscr{A}_{\Delta \Gamma_{s}}^{\mu \mu}$ encodes information equivalent to the effective lifetime

$$
\tau_{\mu \mu} \equiv \frac{\int_{0}^{\infty} t\left\langle\Gamma\left(B_{s}(t) \rightarrow \mu^{+} \mu^{-}\right)\right\rangle d t}{\int_{0}^{\infty}\left\langle\Gamma\left(B_{s}(t) \rightarrow \mu^{+} \mu^{-}\right)\right\rangle d t}=\left\{\begin{array}{l}
(2.04 \pm 0.44(\text { stat }) \pm 0.05(\text { syst })) \text { ps (LHCb [9]) } \\
\left(1.70_{-0.44}^{+0.61}\right) \text { ps (CMS [10]) }
\end{array}\right.
$$

which was firstly measured by the LHCb and CMS collaborations in recent pioneering analyses. For a detailed overview of these experimental studies, see Ref. [11].

In order to probe NP effects through the measured $B_{s}^{0} \rightarrow \mu^{+} \mu^{-}$branching ratio, the quantity

$$
\bar{R}_{\mu \mu}^{s} \equiv \overline{\mathscr{B}}\left(B_{s} \rightarrow \mu^{+} \mu^{-}\right) / \overline{\mathscr{B}}\left(B_{s} \rightarrow \mu^{+} \mu^{-}\right)_{\mathrm{SM}}=0.82 \pm 0.13
$$

plays a central role [4, 5]. Assuming real coefficients $P_{\mu \mu}$ and $S_{\mu \mu}$, we obtain the constraints shown in Fig. 4. Interestingly, $\bar{R}_{\mu \mu}^{s}$ alone does not allow a separation of these contributions and sizeable NP effects could still be present [1]. They could be revealed through a future measurement of $\mathscr{A}_{\Delta \Gamma_{s}}^{\mu \mu}$. Unfortunately, the current experimental values corresponding to (3.4) do not yet have an impact.

Let us now explore implications of these NP constraints for other $B_{q} \rightarrow \ell^{+} \ell^{-}$processes [1]. To this end, we employ a scenario with flavour-universal NP (FUNP) contributions, which is characterised by the feature that the Wilson coefficients $C_{10}^{\ell\left(^{\prime}\right)}, C_{P}^{\ell \ell\left({ }^{\prime}\right)}, C_{S}^{\ell\left(^{\prime}\right)}$ do not depend on flavour labels. In Fig. 5, the corresponding strategy is illustrated in a flowchart.

In the case of $B_{d}^{0} \rightarrow \mu^{+} \mu^{-}$, the ratio

$$
\frac{\overline{\mathscr{B}}\left(B_{d} \rightarrow \mu^{+} \mu^{-}\right)}{\overline{\mathscr{B}}\left(B_{s} \rightarrow \mu^{+} \mu^{-}\right)} \propto\left[\frac{\left|P_{\mu \mu}^{d}\right|^{2}+\left|S_{\mu \mu}^{d}\right|^{2}}{\left|P_{\mu \mu}^{s}\right|^{2}+\left|S_{\mu \mu}^{s}\right|^{2}}\right]\left(\frac{f_{B_{d}}}{f_{B_{s}}}\right)^{2}\left|\frac{V_{t d}}{V_{t s}}\right|^{2}
$$




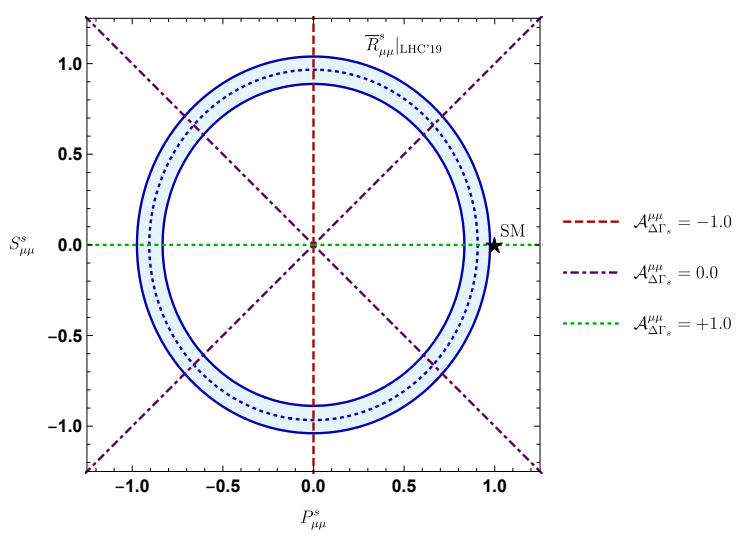

Figure 4: Constraints in the $P_{\mu \mu}^{s}-S_{\mu \mu}^{s}$ plane following from the LHC data and impact of $\mathscr{A}_{\Delta \Gamma_{s}}^{\mu \mu}$ (see Ref. [1]).

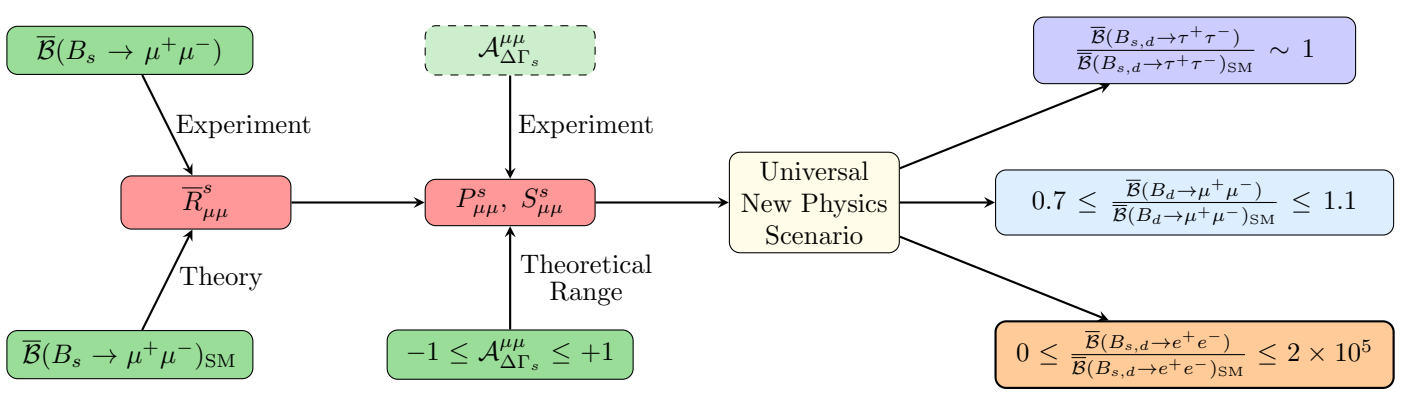

Figure 5: Flowchart to explore the impact of $B_{s} \rightarrow \mu^{+} \mu^{-}$NP constraints for other $B_{s, d} \rightarrow \ell^{+} \ell^{-}$decays.

is a particularly interesting quantity, where the ratio of CKM matrix elements can be determined from an analysis of the unitarity triangle. In the FUNP scenario, an essentially linear correlation between the branching ratios arises, with a moderate suppression of $\overline{\mathscr{B}}\left(B_{d} \rightarrow \mu^{+} \mu^{-}\right)$with respect to the SM expectation, in analogy to the current LHC data for $B_{s} \rightarrow \mu^{+} \mu^{-}$.

Concerning $B_{q}^{0} \rightarrow \tau^{+} \tau^{-}$decays, the NP effects are strongly suppressed by the mass ratio $m_{\mu} / m_{\tau} \sim 0.06$ in the FUNP scenario, resulting in

$$
0.8 \leq \bar{R}_{\tau \tau}^{s} \equiv \overline{\mathscr{B}}\left(B_{s} \rightarrow \tau^{+} \tau^{-}\right) / \overline{\mathscr{B}}\left(B_{s} \rightarrow \tau^{+} \tau^{-}\right)_{\mathrm{SM}} \leq 1.0, \quad 0.995 \leq \mathscr{A}_{\Delta \Gamma_{s}}^{\tau \tau} \leq 1.000,
$$

with a similar picture for $B_{d}^{0} \rightarrow \tau^{+} \tau^{-}$. First experimental bounds were obtained by LHCb [12].

In the case of $B_{q}^{0} \rightarrow e^{+} e^{-}$, we have a situation complementary to $B_{q}^{0} \rightarrow \tau^{+} \tau^{-}$within the FUNP framework, where the NP effects are hugely amplified by the mass ratio $m_{\mu} / m_{e} \sim 207$. In this scenario, the (pseudo)-scalar NP contributions lift the helicity suppression of the extremely small SM branching ratio, as illustrated in Fig. 6, where the red and green bands describe $P_{\mu \mu}^{s}<0$ and $P_{\mu \mu}^{s}>0$, respectively. These results correspond to

$$
0 \leq \bar{R}_{e e}^{s} \leq 1.7 \times 10^{5}, \quad 0 \leq \overline{\mathscr{B}}\left(B_{s} \rightarrow e^{+} e^{-}\right) \leq 1.4 \times 10^{-8} ;
$$

a similar picture arises for the $B_{d} \rightarrow e^{+} e^{-}$decay, with $0 \leq \overline{\mathscr{B}}\left(B_{d} \rightarrow e^{+} e^{-}\right) \leq 3.9 \times 10^{-10}$. The CDF collaboration obtained experimental constraints on these channels back in 2009 [13], finding the upper bounds $\overline{\mathscr{B}}\left(B_{s} \rightarrow e^{+} e^{-}\right)<2.8 \times 10^{-7}$ and $\overline{\mathscr{B}}\left(B_{d} \rightarrow e^{+} e^{-}\right)<8.3 \times 10^{-8}$ (90\% C.L.). In spring 


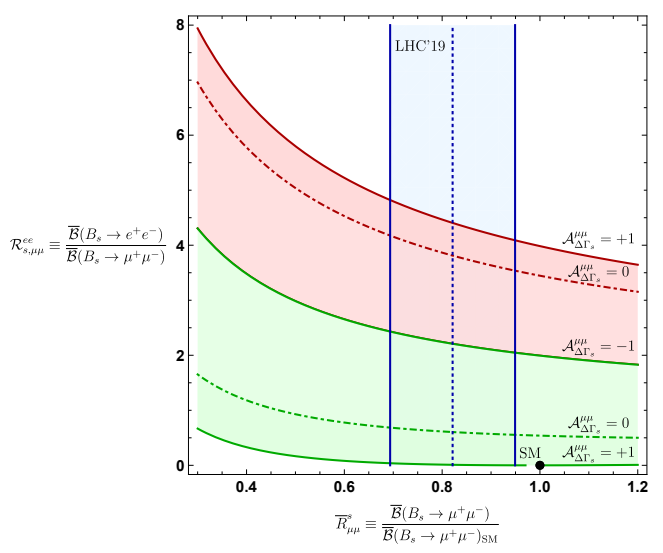

Figure 6: Correlation between the $B_{s} \rightarrow e^{+} e^{-}$and $B_{s} \rightarrow \mu^{+} \mu^{-}$branching ratios in the FUNP scenario.

2020, the LHCb collaboration has reported the first upper bound on $B_{s} \rightarrow e^{+} e^{-}$at the LHC [14]. Assuming no contribution from $B_{d} \rightarrow e^{+} e^{-}$, the constraint $\overline{\mathscr{B}}\left(B_{s} \rightarrow e^{+} e^{-}\right)<9.4(11.2) \times 10^{-9}$ emerges at 90 (95)\% C.L., thereby representing an impressive improvement with respect to the previous bound, putting $B_{s} \rightarrow e^{+} e^{-}$into the interesting regime in Fig. 6. A future observation of this channel would give us an unambiguous signal for physics beyond the SM.

\section{Impact of CP-Violating Phases}

New sources of CP violation may enter through phases of the short-distance coefficients. In the case of $B_{s} \rightarrow \mu^{+} \mu^{-}$decays, we have the following time-dependent CP asymmetry [4, 5]:

$$
\frac{\Gamma\left(B_{s}^{0}(t) \rightarrow \mu_{\lambda}^{+} \mu_{\lambda}^{-}\right)-\Gamma\left(\bar{B}_{s}^{0}(t) \rightarrow \mu_{\lambda}^{+} \mu_{\lambda}^{-}\right)}{\Gamma\left(B_{s}^{0}(t) \rightarrow \mu_{\lambda}^{+} \mu_{\lambda}^{-}\right)+\Gamma\left(\bar{B}_{s}^{0}(t) \rightarrow \mu_{\lambda}^{+} \mu_{\lambda}^{-}\right)}=\frac{\mathscr{C}_{\mu \mu}^{\lambda} \cos \left(\Delta M_{s} t\right)+\mathscr{S}_{\mu \mu} \sin \left(\Delta M_{s} t\right)}{\cosh \left(y_{s} t / \tau_{B_{s}}\right)+\mathscr{A}_{\Delta \Gamma_{s}}^{\mu \mu} \sinh \left(y_{s} t / \tau_{B_{s}}\right)},
$$

where $\lambda$ is the muon helicity, $y_{s}$ was introduced in Eq. (3.1), and

$$
\begin{gathered}
\mathscr{C}_{\mu \mu}^{\lambda}=-\eta_{\lambda}\left[\frac{2|P S| \cos \left(\varphi_{P}-\varphi_{S}\right)}{|P|^{2}+|S|^{2}}\right] \equiv-\eta_{\lambda} \mathscr{C}_{\mu \mu} \\
\mathscr{S}_{\mu \mu}^{\lambda}=\frac{|P|^{2} \sin \left(2 \varphi_{P}-\phi_{S}^{\mathrm{NP}}\right)-|S|^{2} \sin \left(2 \varphi_{S}-\phi_{S}^{\mathrm{NP}}\right)}{|P|^{2}+|S|^{2}} \equiv \mathscr{S}_{\mu \mu}
\end{gathered}
$$

with $\eta_{\mathrm{L}}=+1$ and $\eta_{\mathrm{R}}=-1$, as well as $P \equiv P_{\mu \mu}^{s}, S \equiv S_{\mu \mu}^{s}$. The observable $\mathscr{A}_{\Delta \Gamma_{s}}^{\mu \mu}$ was introduced in Eq. (3.3). The $\mathscr{C}_{\mu \mu}^{\lambda}$ term cancels in the helicity-averaged rates, yielding the $\mathrm{CP}$ asymmetry

$$
\frac{\Gamma\left(B_{s}^{0}(t) \rightarrow \mu^{+} \mu^{-}\right)-\Gamma\left(\bar{B}_{s}^{0}(t) \rightarrow \mu^{+} \mu^{-}\right)}{\Gamma\left(B_{s}^{0}(t) \rightarrow \mu^{+} \mu^{-}\right)+\Gamma\left(\bar{B}_{s}^{0}(t) \rightarrow \mu^{+} \mu^{-}\right)}=\frac{\mathscr{S}_{\mu \mu} \sin \left(\Delta M_{s} t\right)}{\cosh \left(y_{s} t / \tau_{B_{s}}\right)+\mathscr{A}_{\Delta \Gamma_{s}}^{\mu \mu} \sinh \left(y_{s} t / \tau_{B_{s}}\right)} .
$$

It should be noted that the $\mathrm{CP}$ asymmetries satisfy the relation

$$
\left(\mathscr{C}_{\mu \mu}^{\lambda}\right)^{2}+\left(\mathscr{S}_{\mu \mu}\right)^{2}+\left(\mathscr{A}_{\Delta \Gamma_{s}}^{\mu \mu}\right)^{2}=1 .
$$

Consequently, using in addition the ratio $\bar{R} \equiv \bar{R}_{\mu \mu}^{s}$, we have three independent observables which depend on the four NP parameters, $|P|,|S|, \varphi_{P}$ and $\varphi_{S}$. In Ref. [5], the CP asymmetries introduced 


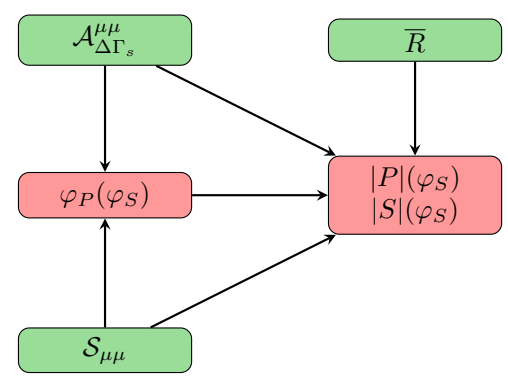

Figure 7: Flowchart for the determination of $\varphi_{P}$ and $|P|,|S|$ as functions of $\varphi_{S}$.
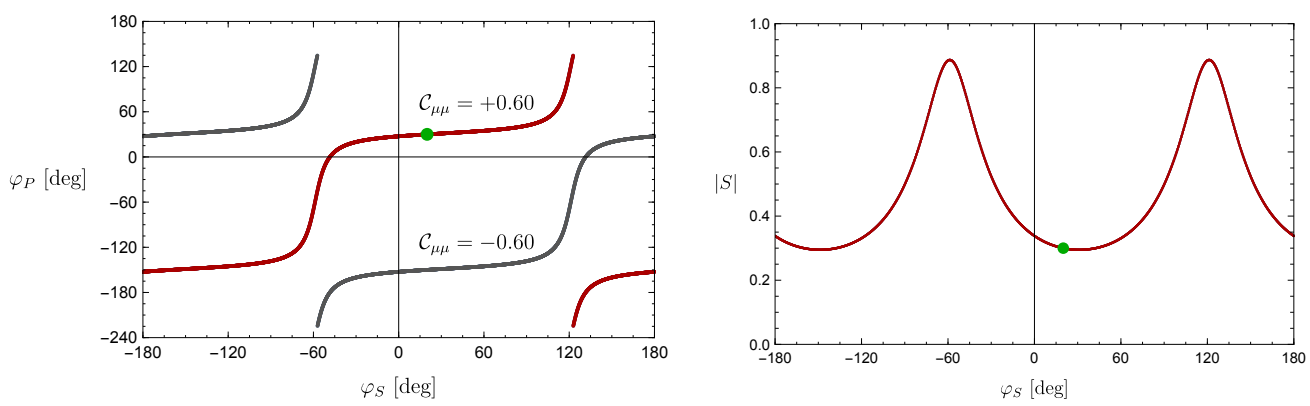

Figure 8: Illustration of the strategy in Fig. 7 for the example of observables given in the text.

above were analysed within specific NP models. A detailed study to probe possible CP-violating phases of $P$ and $S$ was performed in Ref. [2]. The measurement of the $B_{s} \rightarrow \mu^{+} \mu^{-}$observables would allow the extraction of the short-distance coefficients as functions of $\varphi_{S}$ (see Fig. 7). In order to illustrate this feature, we consider a specific example, which is given by the "measured" observables $\bar{R}=0.84, \mathscr{A}_{\Delta \Gamma_{s}}^{\mu \mu}=0.37, \mathscr{S}_{\mu \mu}=0.71$ and $\mathscr{C}_{\mu \mu}=0.60$, yielding the correlations shown in Fig. 8. We observe that we could establish non-vanishing (pseudo)-scalar NP contributions in such a situation, although we could not determine the four NP parameters due to the lack of independent observables. Using more information and assumptions, such as relations arising in the "SM Effective Field Theory" (SMEFT), sharper pictures can be obtained for the NP parameters and their CP-violating phases, as studied in detail in Ref. [2]. The corresponding $B_{s} \rightarrow \mu^{+} \mu^{-}$ measurements offer an exciting playground for the LHCb upgrade and beyond. Detailed feasibility studies would be very desirable. Explorations of CP violation offer valuable insights and are an essential part for revealing the full dynamics of the $B_{s}^{0} \rightarrow \mu^{+} \mu^{-}$decays.

\section{Charged Leptonic Decays}

\subsection{General Features}

Let us now have a closer look at leptonic decays of charged $B$ mesons, following closely Ref. [3], putting $b \rightarrow u \ell \bar{v}_{\ell}$ quark-level transitions into the focus. In the SM, these modes originate from the charged-current tree topology shown in Fig. 9. Using the language of effective field theory, 


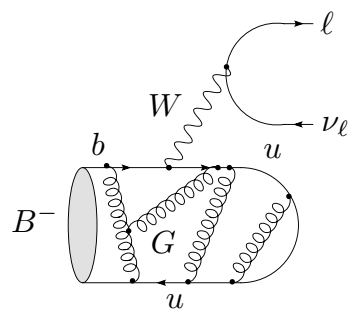

Figure 9: SM contribution to leptonic $B^{-} \rightarrow \ell^{-} \bar{v}_{\ell}$ decays.

the corresponding four-fermion operator is given by

$$
\mathscr{O}_{V_{L}}^{\ell}=\left(\bar{q} \gamma^{\mu} P_{L} b\right)\left(\bar{\ell} \gamma_{\mu} P_{L} v_{\ell}\right)
$$

Beyond the SM, considering (pseudo)-scalar NP operators, yields the low-energy effective Hamiltonian

$$
\mathscr{H}_{\mathrm{eff}}=\frac{4 G_{\mathrm{F}}}{\sqrt{2}} V_{q b}\left[C_{V_{L}} \mathscr{O}_{V_{L}}^{\ell}+C_{S}^{\ell} \mathscr{O}_{S}^{\ell}+C_{P}^{\ell} \mathscr{O}_{P}^{\ell}\right]+\text { h.c. }
$$

with

$$
\mathscr{O}_{S}^{\ell}=(\bar{q} b)\left(\bar{\ell} P_{L} v_{\ell}\right), \quad \mathscr{O}_{P}^{\ell}=\left(\bar{q} \gamma_{5} b\right)\left(\bar{\ell} P_{L} v_{\ell}\right) .
$$

A specific NP scenario of this kind is given by type-II Two-Higgs-Doublet-Models (2HDM), where

$$
C_{P}^{\ell}=C_{S}^{\ell}=-\tan ^{2} \beta\left(\frac{m_{b} m_{\ell}}{M_{H^{ \pm}}^{2}}\right) .
$$

In general, also $\mathscr{O}_{V_{R}}^{\ell}=\left(\bar{q} \gamma^{\mu} P_{R} b\right)\left(\bar{\ell} \gamma_{\mu} P_{L} v_{\ell}\right)$ and $\mathscr{O}_{T}^{\ell}=\left(\bar{q} \sigma^{\mu v} P_{L} b\right)\left(\bar{\ell} \sigma_{\mu v} P_{L} v_{\ell}\right)$ operators may contribute. However, such operators are not considered here. We assume also first that the Wilson coefficients in Eq. (5.2) are real, i.e. do not involve CP-violating phases.

\subsection{Branching Ratios}

In analogy to the leptonic neutral $\bar{B}_{q}^{0} \rightarrow \ell^{+} \ell^{-}$decays, the branching ratios of the charged $B^{-} \rightarrow \ell^{-} \bar{v}_{\ell}$ modes involve only one non-perturbative parameter, the decay constant $f_{B^{-}}$, and are helicity-suppressed in the SM:

$$
\left.\mathscr{B}\left(B^{-} \rightarrow \ell^{-} \bar{v}_{\ell}\right)\right|_{\mathrm{SM}}=\frac{G_{\mathrm{F}}^{2}}{8 \pi}\left|V_{u b}\right|^{2} M_{B^{-}} m_{\ell}^{2}\left(1-\frac{m_{\ell}^{2}}{M_{B^{-}}^{2}}\right)^{2} f_{B^{-}}^{2} \tau_{B^{-}} .
$$

Interestingly, pseudo-scalar operator can lift the helicity suppression:

$$
\mathscr{B}\left(B^{-} \rightarrow \ell^{-} \bar{v}_{\ell}\right)=\left.\mathscr{B}\left(B^{-} \rightarrow \ell^{-} \bar{v}_{\ell}\right)\right|_{\mathrm{SM}}\left|1+\frac{M_{B^{-}}^{2}}{m_{\ell}\left(m_{b}+m_{u}\right)} C_{P}^{\ell}\right|^{2}
$$

In order to obtain constraints from such branching ratios on NP contributions, it is essential to consider clean ratios where the CKM matrix element $\left|V_{u b}\right|$ cancels:

$$
R_{\ell_{2}}^{\ell_{1}} \equiv \frac{m_{\ell_{2}}^{2}}{m_{\ell_{1}}^{2}}\left(\frac{M_{B^{-}}^{2}-m_{\ell_{2}}^{2}}{M_{B^{-}}^{2}-m_{\ell_{1}}^{2}}\right)^{2} \frac{\mathscr{B}\left(B^{-} \rightarrow \ell_{1}^{-} \bar{v}_{\ell_{1}}\right)}{\mathscr{B}\left(B^{-} \rightarrow \ell_{2}^{-} \bar{v}_{\ell_{2}}\right)}=\left|\frac{1+\mathscr{C}_{\ell_{1} ; P}}{1+\mathscr{C}_{\ell_{2} ; P}}\right|^{2},
$$



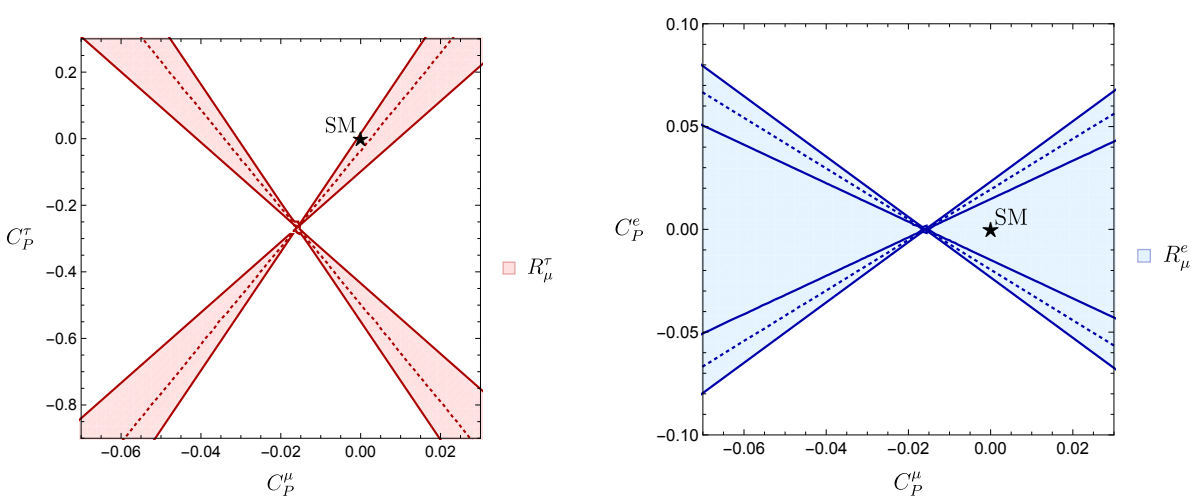

Figure 10: Constraints in the $C_{P}^{\mu}-C_{P}^{\tau}$ and $C_{P}^{\mu}-C_{P}^{e}$ planes following from current data, as discussed in the text.

with

$$
\mathscr{C}_{\ell ; P} \equiv\left|\mathscr{C}_{\ell ; P}\right| e^{i \phi_{\ell}}=\left[\frac{M_{B^{-}}^{2}}{m_{\ell}\left(m_{b}+m_{q}\right)}\right] C_{P}^{\ell} .
$$

The currently available experimental data yield

$$
R_{\mu}^{\tau}=0.76 \pm 0.36, \quad R_{\mu}^{e}<6.48 \times 10^{4},
$$

resulting in the constraints shown in Fig. 10.

\subsection{CP-Violating Phases}

Let us know allow for CP-violating phases of the Wilson coefficients. As we are dealing with charged $B$ decays, we may only have direct $\mathrm{CP}$ violation arising directly at the decay amplitude level. However, the corresponding $\mathrm{CP}$ asymmetries

$$
a_{\mathrm{CP}} \equiv \frac{\mathscr{B}(\bar{B} \rightarrow \bar{f})-\mathscr{B}(B \rightarrow f)}{\mathscr{B}(\bar{B} \rightarrow \bar{f})+\mathscr{B}(B \rightarrow f)}
$$

vanish in leptonic decays at leading order in weak interactions, while higher-order-effects can only generate negligible effects. Consequently, CP-violating NP phases would not be signalled by nonzero values of such $\mathrm{CP}$ asymmetries. In order to explore the impact of such phases, the NP regions in the $\phi_{P}^{\mu}-\left|C_{P}^{\mu}\right|$ plane were studied in Ref. [3], assuming flavour universality for the pseudo-scalar Wilson coefficients of the $\mu, \tau$ and $\mu, e$ systems. The resulting constraints are shown in Fig. 11 .

\subsection{Further Constraints: Semi-Leptonic Decays}

In order to further narrow down the allowed regions in the parameter space of the NP shortdistance coefficients, it is very powerful to use information from semi-leptonic $B$-meson decays. Also in this case, we introduce ratios of branching ratios that are independent of $\left|V_{u b}\right|$ :

$$
\mathscr{R}_{e ; \pi}^{e} \equiv \frac{\mathscr{B}\left(B^{-} \rightarrow e \bar{v}_{e}\right)}{\mathscr{B}\left(\bar{B} \rightarrow \pi e^{-} \bar{v}_{e}\right)}, \quad \mathscr{R}_{\mu ; \pi}^{\mu} \equiv \frac{\mathscr{B}\left(B^{-} \rightarrow \mu^{-} \bar{v}_{\mu}\right)}{\mathscr{B}\left(\bar{B} \rightarrow \pi \mu^{-} \bar{v}_{\mu}\right)}, \quad \mathscr{R}_{\tau ; \pi}^{\tau} \equiv \frac{\mathscr{B}\left(B^{-} \rightarrow \tau^{-} \bar{v}_{\tau}\right)}{\mathscr{B}\left(\bar{B} \rightarrow \pi \tau^{-} \bar{v}_{\tau}\right)}
$$

As discussed in detail in Ref. [3] and shown in Fig. 12, the current data for these quantities can be converted into constraints for the Wilson coefficients. In these studies, interesting subtleties arise 

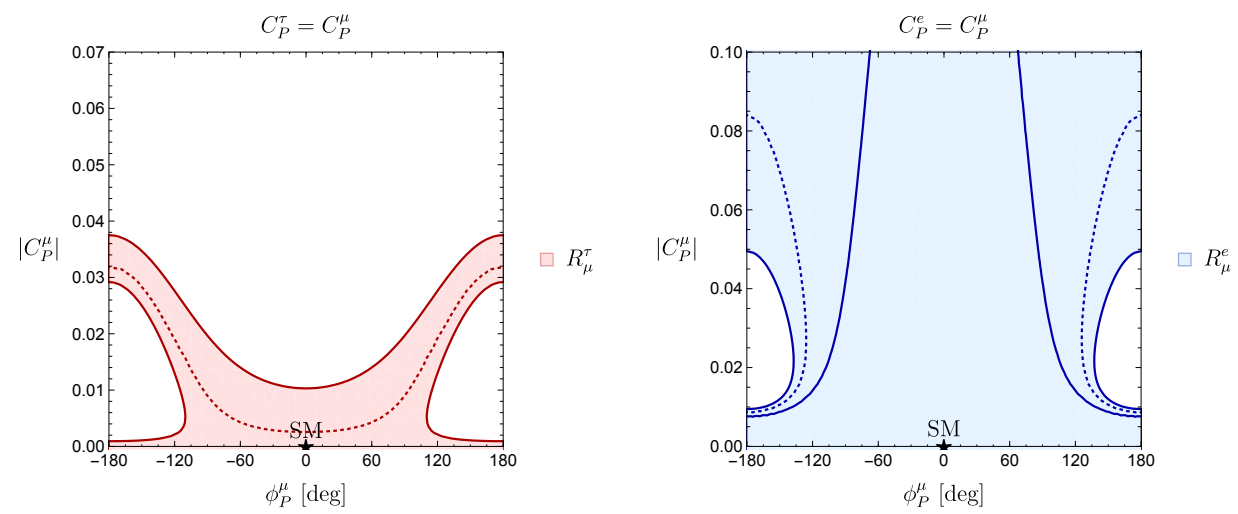

Figure 11: Constraints in the $\phi_{P}^{\mu}-\left|C_{P}^{\mu}\right|$ plane, assuming flavour-universal pseudo-scalar Wilson coefficients.
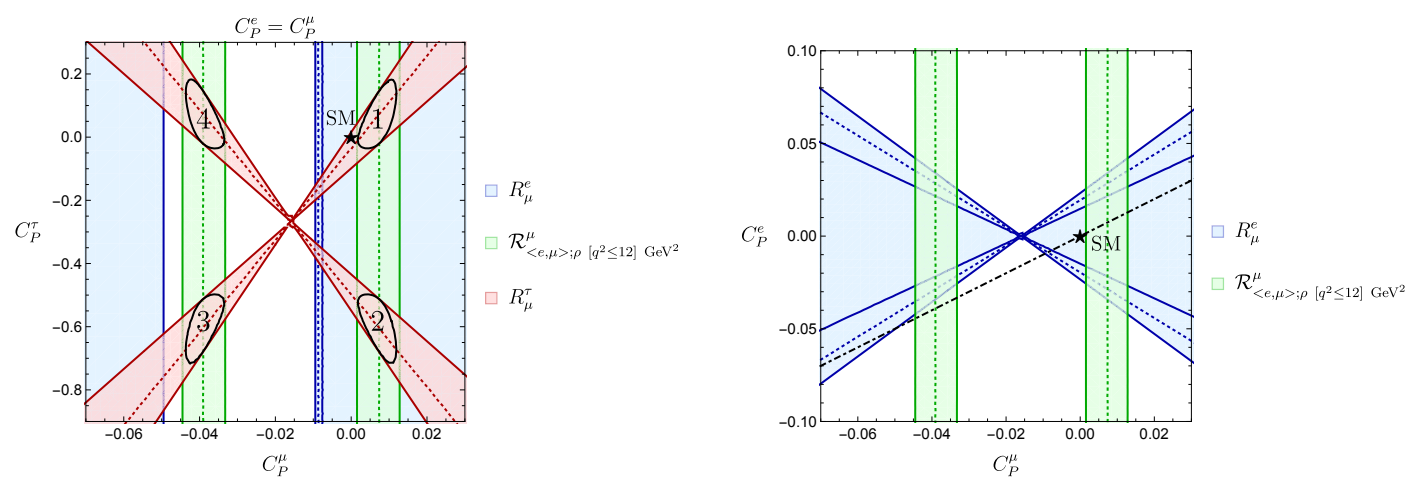

Figure 12: Constraints in the $C_{P}^{\mu}-C_{P}^{\tau}$ and $C_{P}^{\mu}-C_{P}^{e}$ planes following from current data for leptonic (see also Fig. 10) and semi-leptonic $B$ decays.

and new strategies for the determination of $\left|V_{u b}\right|$ and predictions of unmeasured observables in the presence of NP contributions were made, as illustrated in Fig. 13. Interestingly, pseudo-scalar $\mathrm{NP}$ effects may lift the helicity suppression of the $B^{-} \rightarrow e^{-} v_{e}$ mode (in analogy to $\bar{B}_{s, d}^{0} \rightarrow e^{+} e^{-}$ discussed above), as shown in Fig. 14. It will be important to search for this channel in the future data taking at the high-precision frontier, pushing the limits to unexplored new territory.

The studies using leptonic and semi-leptonic $B$ decays discussed above, allowing us to probe possible violations of lepton flavour universality, can be nicely complemented through similar analyses in the charm sector, as discussed in detail in a recent analysis [15].

\section{Conclusions and Outlook}

We are moving towards new frontiers with leptonic $B$ decays. Concerning the neutral leptonic $B_{s, d} \rightarrow \ell^{+} \ell^{-}$modes, the decay width difference $\Delta \Gamma_{s}$ provides access to another (theoretically clean) observable $\mathscr{A}_{\Delta \Gamma_{s}}^{\mu \mu}$. The LHCb and CMS collaboration have recently performed pioneering measurements of the effective lifetime of $B_{s} \rightarrow \mu^{+} \mu^{-}$, providing information equivalent to $\mathscr{A}_{\Delta \Gamma_{s}}^{\mu \mu}$. It will be important to get a sharper picture in the future. We have seen that $\overline{\mathscr{B}}\left(B_{s} \rightarrow e^{+} e^{-}\right)$could be hugely enhanced through NP effects, even to the $\overline{\mathscr{B}}\left(B_{s} \rightarrow \mu^{+} \mu^{-}\right)$regime. LHCb has recently reported a first limit of $9 \times 10^{-9}(90 \%$ C.L.), superseding results by the CDF collaboration from 2009. It 


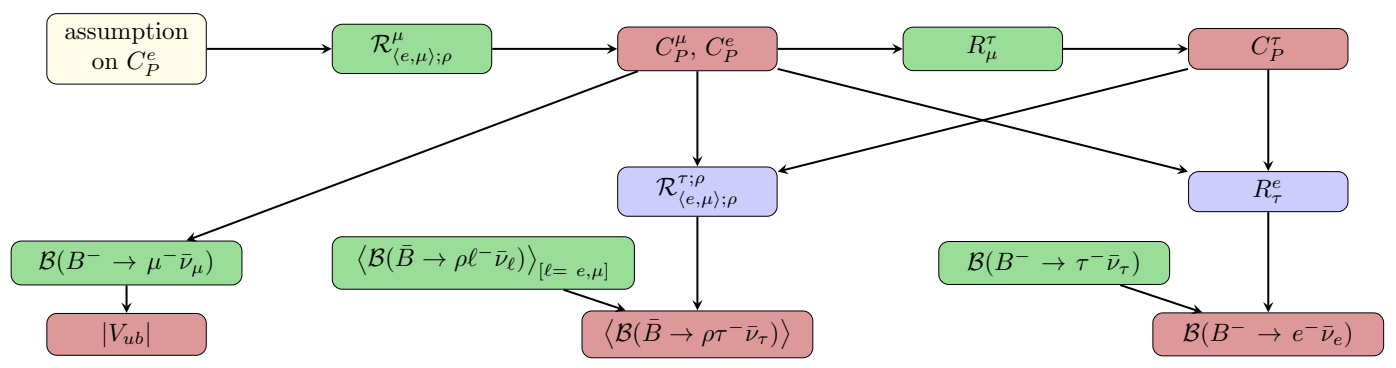

Figure 13: New strategy to extract $\left|V_{u b}\right|$ in the presence of NP and to predict yet unmeasured observables.

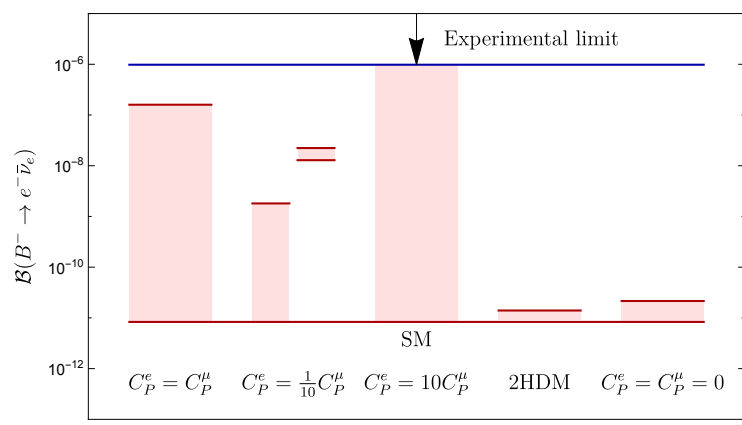

Figure 14: Illustration of the lift of the helicity suppression of the $B^{-} \rightarrow e^{-} v_{e}$ decay.

will be exciting to see much stronger constraints in the future. An observation of the $B_{s(d)} \rightarrow e^{+} e^{-}$ modes would give a clear NP signal. The $B_{s, d} \rightarrow \ell^{+} \ell^{-}$modes provide also interesting strategies for revealing new sources of $\mathrm{CP}$ violation.

Charged leptonic $B^{-} \rightarrow \ell^{-} \bar{v}_{\ell}$ decays decays offer sensitive probes of lepton flavour universality in a clean setting, showing also a powerful interplay with semi-leptonic decays such as $B \rightarrow \rho \ell \bar{v}_{\ell}$ and $B \rightarrow \pi \ell \bar{v}_{\ell}$. Since $\mathscr{B}\left(B^{-} \rightarrow e^{-} \bar{v}_{e}\right)$ could be hugely enhanced through NP effects, it is important to search for this channel at Belle II. An observation would give us clear evidence for physics beyond the SM.

Leptonic rare decays of $B$ mesons continue to offer an interesting laboratory for to "stress test" the SM with unprecedented precision and to explore scenarios of new interactions and particles. The future high-precision era of flavour physics with valuable and rich data from Belle II, the LHC upgrade and beyond will be very exciting!

\section{Acknowledgments}

I am very grateful to Gilberto Tetlalmatzi-Xolocotzi, Ruben Jaarsma, Giovanni Banelli and Daniela Galárraga Espinosa for our collaborations on topics discussed here. I would also like to thank George Zoupanos and his co-organisers for creating such an interesting and most enjoyable meeting on Corfu, allowing also many junior physicists to attend. This research has been supported by the Netherlands Foundation for Fundamental Research of Matter (FOM) programme 156, "Higgs as Probe and Portal", and by the National Organisation for Scientific Research (NWO). 


\section{References}

[1] R. Fleischer, R. Jaarsma and G. Tetlalmatzi-Xolocotzi, JHEP 1705, 156 (2017).

[2] R. Fleischer, D. Galárraga Espinosa, R. Jaarsma and G. Tetlalmatzi-Xolocotzi, Eur. Phys. J. C 78, 1 (2018).

[3] G. Banelli, R. Fleischer, R. Jaarsma and G. Tetlalmatzi-Xolocotzi, Eur. Phys. J. C 78 (2018) no.11, 911; SciPost Phys. Proc. 1, 013 (2019).

[4] K. De Bruyn, R. Fleischer, R. Knegjens, P. Koppenburg, M. Merk, A. Pellegrino and N. Tuning, Phys. Rev. Lett. 109, 041801 (2012).

[5] A. J. Buras, R. Fleischer, J. Girrbach and R. Knegjens, JHEP 1307, 77 (2013).

[6] K. De Bruyn, R. Fleischer, R. Knegjens, P. Koppenburg, M. Merk and N. Tuning, Phys. Rev. D 86, 014027 (2012).

[7] K. De Bruyn and R. Fleischer, JHEP 1503, 145 (2015).

[8] M. Beneke, C. Bobeth and R. Szafron, Phys. Rev. Lett. 120, 011801 (2018)

[9] R. Aaij et al. [LHCb Collaboration], Phys. Rev. Lett. 118, 191801 (2017).

[10] A. M. Sirunyan et al. [CMS Collaboration], arXiv:1910.12127 [hep-ex].

[11] M. Pepe-Altarelli, these proceedings.

[12] R. Aaij et al. [LHCb Collaboration], Phys. Rev. Lett. 118, 251802 (2017).

[13] T. Aaltonen et al. [CDF Collaboration], Phys. Rev. Lett. 102, 201801 (2009).

[14] R. Aaij et al. [LHCb Collaboration], arXiv:2003.03999 [hep-ex].

[15] R. Fleischer, R. Jaarsma and G. Koole, Eur. Phys. J. C 80, 153 (2020). 